\title{
Clinico-Pathological Study of Invasive Fungal Sinusitis in Ibadan, Nigeria: A Case Series
}

\author{
Oluyemi Fasina1* ${ }^{*}$ Adekunle Daniel², Gabriel Olabiyi Ogun³, John A. Babarinde² \\ ${ }^{1}$ Department of Ophthalmology, University College Hospital, University of Ibadan, Ibadan, Nigeria \\ ${ }^{2}$ Department of Otorhinolaryngology, University College Hospital, University of Ibadan, Ibadan, Nigeria \\ ${ }^{3}$ Pathology Department, University College Hospital, University of Ibadan, Ibadan, Nigeria \\ Email: *yemifash2000@yahoo.com
}

How to cite this paper: Fasina, O., Daniel, A., Ogun, G.O. and Babarinde, J.A. (2020) Clinico-Pathological Study of Invasive Fungal Sinusitis in Ibadan, Nigeria: A Case Series. Case Reports in Clinical Medicine, 9, 87-95.

https://doi.org/10.4236/crcm.2020.94014

Received: March 10, 2020

Accepted: April 6, 2020

Published: April 9, 2020

Copyright (c) 2020 by author(s) and Scientific Research Publishing Inc. This work is licensed under the Creative Commons Attribution International License (CC BY 4.0).

http://creativecommons.org/licenses/by/4.0/

\begin{abstract}
Background: Invasive fungal sinusitis is one of the less common forms of fungal infection more commonly described in North Africa and Asia. It affects healthy and immunologically competent individuals typically complicating chronic rhinosinusitis. Surgical debridement and adjuvant antifungal treatment are the mainstay of management of this condition. Objective: To describe the clinical presentation, pathological features and management of patients with invasive fungal sinusitis managed at a tertiary health facility, southwestern Nigeria. Method: Medical records of all patients with invasive fungal sinusitis managed between January 2009 and December 2018 were retrospectively reviewed. Results: Six patients with invasive fungal sinusitis were managed during the study period. All patients were immunocompetent and the mean age at presentation was 30.7 years \pm 7.2 years. The average duration of symptoms prior to presentation was 18.5 months (Interquartile range, 67.5 months), and all six patients had orbital involvement and intracranial extension of the mass at presentation. They all had surgical debridement, and the specimens were sent for histological examination. Mycological studies carried out on two specimens isolated Aspergillus fumigatusas the fungal agent and all patients were started on post-operative oral antifungal chemotherapy. Five patients completed their chemotherapy and fared well with no recurrence while one patient had irregular and incomplete antifungal treatment, and developed recurrent infection before abandoning further treatment. Conclusion: Chronic invasive fungal sinusitis is an uncommon infection and fairly difficult to manage. However, timely diagnosis and combined surgical and medical treatment can give good outcomes in the patients.
\end{abstract}

\section{Keywords}

Clinico-Pathological Features, Ibadan, Immunocompetent, Invasive Fungal 


\section{Introduction}

Fungal sinusitis, broadly classified as invasive or non-invasive, is an uncommon infection, but there has been an increase in its incidence in the past few decades. Invasive fungal sinusitis can be acute or chronic in presentation. Chronic invasive fungal sinusitis (CFS), unlike acute invasive fungal sinusitis, occurs in immunocompetent, non-atopic patients who often have a history of chronic rhinosinusitis. It runs an indolent course and frequently extends beyond the sinus walls thus mimicking locally aggressive neoplasms [1] [2]. Initially asymptomatic, CFS can later present with pain, chronic headache, proptosis and cranial nerve deficits from invasion of adjacent structures. [1] Mycological studies had demonstrated majorly Aspergillus and a few Dermatiaceous species in the aetiology of the disease. [3] [4] Computed tomogram and magnetic resonance imaging are typically used to assess the extent of involvement of the paranasal sinuses and invasion of adjacent structures. Surgical debridement is required to treat this infection with extensive removal of involved tissue and bone. Antifungal chemotherapy also plays a major role in the management of the infective disease. The disease has been reported to be endemic in Sudan [5] and Northern India [4] due to the hot and dry climate in these areas which has been postulated to play a role in the aetiopathogenesis of the infection but has been rarely reported in Nigeria. To the best knowledge of the authors, there are no published reports of chronic invasive fungal sinusitis in our locality, hence this report of the cases managed at a tertiary health facility in southwestern Nigeria.

\section{Method}

Medical records of patients with chronic invasive fungal sinusitis managed over 10 years (January 2009 - December 2018) at a tertiary health facility, southwestern Nigeria were reviewed. Patients' clinical presentation, immune status, radiological imaging, surgical intervention, histological findings as well as treatment and outcome were studied. Diagnosis of chronic invasive fungal sinusitis was made by histopathological examination of all surgical specimens and mycological studies where possible, supported by radiological imaging in the patients. This study adhered to the Tenets of Declaration of Helsinki and informed consent was obtained from each patient for the publication of the case series.

\section{Case Series}

Case 1: A 35-year-old female presented with gradual protrusion of the right eye, associated with loss of vision and pain of 6 years duration. She also had spontaneous, intermittent right epistaxis with bilateral nasal blockage, hyposmia and mucopurulent nasal discharge. There were no ear or throat symptoms and she 
had no features suggestive of immunosuppression. Clinical examination revealed a pale mass completely obstructing right nasal cavity, not bleeding to touch and the ear and throat appeared normal. Ocular examination revealed acuity of hand movement in the right eye, severe non-axial proptosis, and a firm mass in the supero-medial orbit. Computed Tomogram (CT) revealed an isodense mass in the right orbit andright maxillary, ethmoidal and frontal sinuses. There was an extensive aerocele into the left frontal region with direct communication with the nasal cavity. She subsequently underwent right extended lateral rhinotomy, for medial maxillectomy, right orbitotomy and bi-frontal craniotomy for excision of the tumuor and aerocele. Intraoperatively, a firm, well circumscribed irregularly shaped mass extending from the right antrum to the right ethmoid and the orbit, displacing the right eyeball laterally was excised. Histopathological examination of the tissue revealed chronic invasive fungal sinusitis. She was commenced on oral antifungal drug (Voriconazole) post-operatively with regular monitoring of the liver function and she fared well on regular follow up for about six months before she was lost to follow-up.

Case 2: A 24-year-old male presented with purulent left nasal discharge of a year duration and protrusion of the left eyeball of 8 months duration. There was associated nasal obstruction which was initially intermittent but later became persistent with accompanying hyposmia and frontal headache, but no fever. The proptosis was non-axial, progressive, painful and associated reduced vision. There were no features suggestive of immunosuppression in the patient and clinical examination showed reduced patency of the left nasal cavity which was filled with a brownish mass arising from its lateral wall. The mass was not sensitive, and there was no bleeding to touch. Ear and throat examinations were normal. CT scan of paranasal sinuses showed an isodense lesion in left maxillary, ethmoidal and frontal sinuses with orbital and intracranial involvement. $\mathrm{He}$ subsequently had left external frontoethmoidectomy, orbitotomy, and craniotomy for tumor excision. Histopathological examination of the specimen revealed an invasive fungal sinusitis. He was commenced on oral antifungal (ketoconazole) and discharged to Out-patient Clinic. However, he was irregular with the antifungal treatment and follow-up visits and re-presented with recurrent swelling for which a repeat debridement was advised, but he declined and was eventually lost to follow up.

Case 3: A 32-year-old female presented with bilateral nasal discharge of 13 months duration and protrusion of the left eye of 12 months duration. The nasal discharge was mucopurulent, occasionally stained with blood, and later associated with bilateral nasal obstruction, anosmia, snoring, hyponasal speech and frontal headache. There were no otologic or throat symptoms. Proptosis was painless, progressive and associated with loss of vision. She had no features suggestive of immunosuppression. Examination of the nose showed pale glistening mass filling both nasal cavities which appeared to be arising from lateral wall of the nasal cavity, not sensitive to probe and no bleeding to touch. Ear and throat 
examinations were essentially normal. Ocular examination findings showed acuity of no light perception in the left eye with marked non-axial proptosis, chemosis and purulent discharge. CT scan of the paranasal sinuses showed isodense lesion in the left frontal and sphenoidal sinuses with destruction of orbital roof and intracranial extension. She had per nasal biopsy of the intranasal mass for histopathological examination and fungal culture and these confirmed Aspergillus flavus isolate from the tissue. She subsequently had bilateral external frontoethmoidectomy, bilateral intranasal antrostomy, left orbital exenteration and craniotomy with gross total tumor excision Post-operatively, she received oral Itraconazole and had repeated antral lavage with fungusol. She is presently doing well on regular follow up with no evidence of recurrence six months post-operatively.

Case 4: A 42-year-old female presented with painful progressive protrusion of her left eye of 6 months duration associated with excessive tearing. There was no nasal discharge, blockage, epistaxis or anosmia, and no otologic or throat symptoms and clinical examination of the ear, nose and throat was essentially normal. She had no features suggestive of immunosuppression. A firm swelling located in the left aspect of the glabella with inferolateral displaced left proptosis was noted on clinical examination. CT scan showed ill-defined heterogenous mass involving the medial part of the left orbit, anterior ethmoidal cells and frontal sinus, displacing the globe inferolaterally. There was destruction of the skull base with extension of the mass into the anterior cranial fossa. Surgical intervention was delayed for about 15 months due to financial constraints and the lesion had expanded to involve the skin of the forehead with discharging sinus over it. She eventually had bi-frontal craniotomy, external fronto-ethmoidectomy and orbitotomy for tumor excision. Histopathological examination and fungal culture of the specimen confirmed Aspergillus flavusinfection and she was treated with oral voriconazole. She is on regular follow up in stable clinical condition and no evidence of recurrence eight months post-operatively.

Case 5: A 28-year-old male presented with recurrent left nasal blockage and protrusion of the left eye of 8 years duration. The nasal blockage was associated with mucoid discharge, spontaneous epistaxis, and hyposmia. There were no ear or throat symptoms and no features suggestive of immunosuppression. Clinical examination of the nose showed pale intranasal mass in both nasal cavities more on the left with thick mucoid discharge but no bleeding to touch and a firm, non-tender mass lateral to the left nostril extending to the cheek with no skin involvement. Ocular examination showed a left firm, non-tender inferior orbital mass with non-axial proptosis, superolateral displacement of the globe and reduced vision (Figure 1). CT scan showed left inferior orbital mixed density lesion extending to the left maxillary sinus and superiorly into the anterior cranial fossa (Figure 1). He subsequently had left medial maxillectomy, external fronto-ethmoidectomy, orbitotomy and trans-frontal intracranial tumor excision. Histopathological examination showed features of chronic fungal rhinosinusitis 


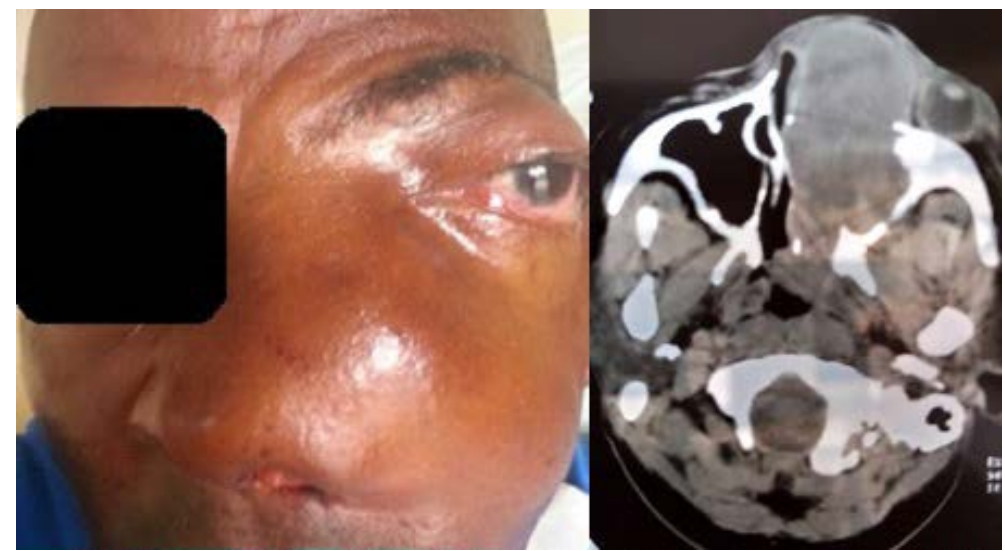

(a)

(b)

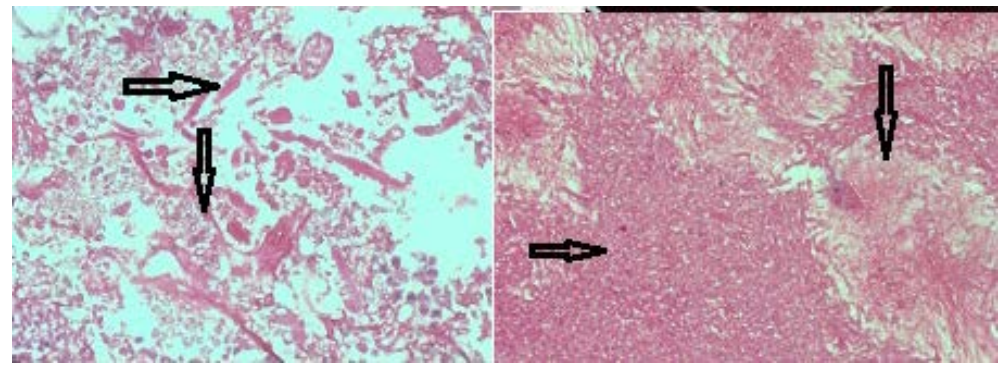

(c)

(d)

Figure 1. (a) Clinical picture of the 28-year-old male with chronic fungal sinusitis involving the paranasal sinuses, orbit and anterior cranial fossa; (b) CT scan picture of the patient showing heterogeneous soft tissue mass involving the left maxillary antrum, ethmoids and the orbit; (c) Photomicrograph of the histopathologic section (Periodic Acid Schiff, $\times 200$ ) of the excised tissue specimen, vertical arrow showing the yeast and horizontal arrow the hyphae; (d) Photomicrograph of the histopathologic section (Haematoxylin and Eosin, $\times 100$ ) of the excised tissue specimen showing the colony of fungus (horizontal arrow) with branching hyphae (vertical arrow).

(Figure 1) and he was treated with oral voriconazole and has fared well six months post-operatively.

Case 6: A 23-year-old man presented with recurrent bilateral nasal blockage of 3 years duration and protrusion of the left eye of 2 years duration. The nasal blockage was initially intermittent, but progressively worsened and associated with thick mucoid discharge and anosmia. There were no ear or throat symptoms and no features suggestive of immunosuppression. The left proptosis was painless, progressive, and associated with reduced vision. Examination of the nasal cavity showed a pale glistening mass filling both nasal cavities with no bleeding to touch. Ear and throat examinations were normal. Ocular examination revealed left non-axial proptosis, and acuity of counting fingers. Examination of the right eye showed normal findings. CT scan of paranasal sinuses showed enhancing soft tissue mass within the left frontal sinus with bone destruction and cavity expansion intracranially, and expansion into the left orbit with non-axial proptosis. There were bone destruction and extension of the mass into the ethmoid and sphenoid sinuses and left nasal cavity. He subsequently 
had tumor excision through bilateral medial maxillectomy, left ethmoidectomy with sphenoidotomy, orbitotomy, and bifrontal craniotomy. Histopathological examination of the specimen revealed fungal-induced chronic rhinosinusitis, and he was treated with oral Itraconazole tablets. He has fared well and presently on regular on follow up Out-patient clinic visits 6 months post-operatively.

\section{Discussion}

Chronic invasive fungal sinusitis is uncommon in our environment. A hospitalbased study [6] on microorganisms aspirated from the maxillary sinus in patients with sinusitis demonstrated the presence of fungi in $14.58 \%$ of patients. The fungi isolated in the study [6] were Aspergillus flavus, Aspergillus fumigatus and Candida albicans. All six cases in our series were healthy individuals without any features of immunosuppression. Chronic invasive fungal sinusitis has been shown to have a clinical course greater than 12 weeks, occurring in immunecompetent individuals, and Aspergillus flavusas the most common fungal agent identified indifference to acute invasive fungal sinusitis which occurs in immunecompromised individuals, and frequently implicated fungal agents include Aspergillus species and fungi in the order of mucorale [7]. Mould-Shalom et al in their study [8] on acute invasive fungal sinusitis reported diabetes mellitus and other immune compromise in their patients.

The mean age of our patients was 30.7 years \pm 7.2 years similar to the report of Alrajhi et al. [9] and an equal gender distribution was also noted in our series (Table 1). Mostly, young, active and immunocompetent individuals of both sexes are affected by this condition [2] [9].

All the patients presented late to our facility (mean duration of symptoms, 38.5 months; range, 6 to 96 months) with orbital and intracranial involvement at presentation (Table 2). A slow progression of this disease has been documented, usually over months to years and patients usually present when the swelling extends to the orbit or base of the skull [1]. The typical presenting symptoms are related to chronic rhinosinusitis, upper respiratory allergies or nasal polyposis. Erosion into the orbit leads to proptosis while skull base involvement can cause chronic headache, seizures, decreased mental status or focal neurological deficits. Extension through the sphenoid sinus can give rise to orbital apex syndrome or cavernous sinus syndrome [10]. Similar to a previous report [1], proptosis was the main ocular presenting complaint in our cases.

All six patients had pre-operative contrast enhanced CT scan, which though not confirmatory, is a useful radio-imaging in defining the extent of the disease. Magnetic resonance imaging is useful in identifying involvement of the dura as well as the extent of intradural disease [2]. Chronic fungal sinusitis typically starts from the maxillary sinus on radio-imaging studies; however, it was not possible to accurately determine the paranasal sinus of origin in our patients as they presented with advanced disease and multiple sinus involvement (Table 2). Differentiating between invasive fungal sinusitis and malignant tumors of the 
Table 1. Age and sex distribution of the patients.

\begin{tabular}{ccc}
\hline Cases & Age (years) & Sex \\
\hline 1 & 35 & Female \\
2 & 24 & Male \\
3 & 32 & Female \\
4 & 42 & Female \\
5 & 28 & Male \\
6 & 23 & Male \\
\hline
\end{tabular}

Table 2. Clinical presentations of the patients.

\begin{tabular}{cccccccc}
\hline Cases & $\begin{array}{c}\text { Duration of } \\
\text { symptoms }\end{array}$ & $\begin{array}{c}\text { Eye } \\
\text { affected }\end{array}$ & Visual loss & $\begin{array}{c}\text { Paranasal sinus } \\
\text { involvement }\end{array}$ & $\begin{array}{c}\text { Nostril } \\
\text { affectation }\end{array}$ & $\begin{array}{c}\text { Intracranial } \\
\text { extension }\end{array}$ & $\begin{array}{c}\text { pollow-up } \\
\text { period }\end{array}$ \\
\hline 1 & 72 months & Right & Yes & Max + Eth + Fr & Right & Yes & 6 months \\
2 & 12 months & Left & Yes & Max + Eth + Fr & Left & Yes & 3 months \\
3 & 13 months & Left & Yes & Fr + Sph & Bilateral & Yes & 6 months \\
4 & 6 months & Left & No & Fr + Eth & Nil & Yes & 8 months \\
5 & 96 months & Left & Yes & Max + Eth + Fr & Bilateral & Yes & 6 months \\
6 & 36 months & Left & Yes & Fr + Eth + Sph & Bilateral & Yes & 6 months \\
\hline
\end{tabular}

Max $=$ maxillary, Eth $=$ ethmoid, $\mathrm{Fr}=$ frontal, $\mathrm{Sph}=$ sphenoid.

paranasal sinuses is difficult both clinically and on radio-imaging, therefore, distinction is usually made onexamination of the tissue specimen at histology. The infective agents can also be identified through culture and microscopic visualization of broad, non-septate hyphae [7] [11] [12], Diagnoses of chronic fungal sinusitis was made in our series by histopathological identification of fungal organism in the tissue specimenin all six cases, and mycological studies in two patients identified Aspergillus flavusas the aetiological agent (Table 3). Aspergillus speciesare the most common agent isolated in chronic fungal sinusitis, and tissue cultures are positive in about $50 \%$ of the cases [7] [11].

Regarding treatment for chronic invasive fungal sinusitis, several authors have advocated combination therapy which includes surgical debridement with adjuvant antifungal therapy. The extent of surgery required to extirpate the disease has however been controversial, but wide aeration of the sinuses and long-term antifungal therapy is advocated [2] [13] [14]. Amphotericin B as well as the Azoles has been used as adjuvant therapy post-surgical debridement. All our patients had wide surgical debridement and the azole group of antifungals as adjuvant therapy (Table 3 ) with good response and satisfactory outcome achieved in five patients who completed their treatment. One of the patients however, was non-compliant with his medication due to financial constraints, had recurrent infection and was eventually lost to follow-up.

Our study is limited in the context that specific fungal identification by culture could not be done in four of the cases due to non-availability of the service in our facility at those periods. 
Table 3. Diagnoses and drug therapy in the patients.

\begin{tabular}{ccc}
\hline Cases & Diagnosis & Drug treatment \\
\hline 1 & Histology & Voriconazole \\
2 & Histology & Ketoconazole \\
3 & Histology + culture & Itraconazole \\
4 & Histology + culture & Voriconazole \\
5 & Histology & Voriconazole \\
6 & Histology & Itraconazole \\
\hline
\end{tabular}

\section{Conclusion}

In conclusion, chronic invasive fungal sinusitis is uncommon in our locality and affects young, active and immunocompetent individuals. Appropriate imaging studies and histopathological examination of excised tissue help in making the diagnosis of this infective disease and satisfactory treatment outcome can be achieved by effective surgical debridement and oral antifungal mediations.

\section{Conflicts of Interest}

The authors declare no conflicts of interest regarding the publication of this paper.

\section{References}

[1] Verass, B., Malik, O.A., el-Tayeb, A.A., el-Daoud, S., Mahgoub, E.S. and el-Hassan, A.M. (1973) Further Observations on the Primary Paranasal Aspergillus Granuloma in Sudan. The American Journal of Tropical Medicine and Hygiene, 22, 765-772. https://doi.org/10.4269/ajtmh.1973.22.765

[2] Washburn, R.G., Kennedy, D.W., Begley, M.G., Henderson, D.K. and Bennet, J.E. (1988) Chronic Fungal Sinusitis in Apparently Normal Hosts. Medicine, 67, 231-247. https://doi.org/10.1097/00005792-198807000-00004

[3] Chakrabarti, A., Sharma, S.C. and Chander, J. (1992) Epidemology and Pathogenesis of Paranasal Sinus Mycosis. Otolaryngology: Head and Neck Surgery, 107, 45-50. https://doi.org/10.1177/019459988910700606.1

[4] Shagar, M.A., Montgomery, W.W. and Hyslop, N.E. (1981) Alternaria Sinusitis. Annals of Otology, Rhinology \& Laryngology, 90, 251-254. https://doi.org/10.1177/000348948109000311

[5] Kurien, M., Anandi, V., Raman, R. and Brahmandathan, K.N. (1992) Maxillary Sinus Fusariosis in Immunocompetent Hosts. The Journal of Laryngology \& Otology, 106, 733-736. https://doi.org/10.1017/S0022215100120729

[6] Fasunla, A.J., Fayemiwo, S.A., Bakare, R.A. and Ijaduola, G.T.A. (2018) Aerobic Bacteria and Fungal Isolates in Maxillary Sinusitis of Adults in a Resource Poor Environment. African Journal of Clinical Experimental Microbiology, 19, 113-120. https://doi.org/10.4314/ajcem.v19i2.6

[7] De Shazo, R.D., Chapin, K. and Swain, R.E. (1997) Fungal Sinusitis. The New England Journal of Medicine, 337, 254-259. https://doi.org/10.1056/NEJM199707243370407 
[8] Mould-Shalom, A.F., Opaluwah, E.A. and Akabe, E.A. (2017) Acute Invasive Fungal Rhinosinusitis with Orbital Abscess (Rhino-Orbital Mucormycosis): A Case Study. Nigerian Journal of Ophthalmology, 25, 152-156.

[9] Alrajhi, A.A., Enani, M., Mahasin, Z. and Al-Oman, K. (2001) Chronic Invasive Aspergillosis of the Paranasal Sinuses in Immunocompetent Hosts from Saudi Arabia. The American Journal of Tropical Medicine and Hygiene, 65, 83-86. https://doi.org/10.4269/ajtmh.2001.65.83

[10] Washburn, R.G. (1998) Fungal Sinusitis. Current Clinical Topics in Infectious Diseases, 18, 60-74.

[11] Thompson, G.R. and Patterson, T.F. (2012) Fungal Disease of the Nose and Paranasal Sinuses. Journal of Allergy and Clinical Immunology, 129, 321-326.

https://doi.org/10.1016/j.jaci.2011.11.039

[12] Chakrabati, A., Denning, D.W., Ferguson, B.J., Ponikau, J., Buzina, W., Kita, H., et al. (2009) Fungal Rhinosinusitis: A Categorization and Definitional Schema Addressing Current Controversies. Laryngoscope, 119, 1809-1818. https://doi.org/10.1002/lary.20520

[13] Sandison, A.T., Gentles, J.C., Davidson, C.M. and Branco, M. (1969) Aspergilloma of the Paranasal Sinuses and Orbit in Northern Sudanese. Sabouraudia, 6, 57-69. https://doi.org/10.1080/00362176885190101

[14] Zieske, L.A., Kopke, R.D., Hamill, R. (1991) Dermatiaceous Fungal Sinusitis. Otolaryngology: Head and Neck Surgery, 105, 567-577.

https://doi.org/10.1177/019459989110500408 